\title{
Population Density, Economic Threshold and Injury Levels of Tetranychus urticae and Petrobia tritici Infesting Wheat Plants at Sharkia and Beheira Governorates, Egypt
}

\author{
Fatma Sh. Kalmosh \\ Plant Protection Research Institute, ARC, Dokki, Giza, Egypt, bkalmosh@yahoo.com.
}

\begin{abstract}
Assessment of the economic damage threshold (ETL) and economic injury level (EIL) of the two tetranychid Tetranychus urticae and Petrobia tritici infested wheat (Giza 168) were studied during 2016/2017 season at Sharkia and Beheira governorates. Also, the population density of both mite species were estimated. Results cleared that the values of (ETL) were less than (EIL) level. The (ETLs) values of T. urticae on wheat plants at Beheira and Sharkia (as x1 \&x2 peaks results) ranged 4-4.33 and 4.33-10 individuals /leaf, while the (EIL)values ranged 4.33-5 and 10.33- 13.33 individuals /leaf in the two localities, respectively. In the same trend the ETLs of P.tritici on wheat plants at Sharkia and Beheira (as $\mathrm{x} 1 \& \mathrm{x} 2$ peaks results) ranged 3.33-4.00 and 3.33 individuals. /leaf, while the EILs ranged 5-5.33 and 3.67-5.33 Individuals / leaf in the two localities, respectively. In addition, the results of population density of the two-mite species showed that, there were two peaks for each species at the two localities. Statistical analysis detected a positive insignificant correlation between the population of the two mite species and average temperature while it was insignificantly negative with average of R.H. \%. The relatively high effect as partial regression (E.V. \%) for tested climatic factors of 5.18\% was recorded for average temperature on P.tritici at Sharkia governorate, while the relatively high multiple correlation for the two tested climatic factors together was $17.07 \%$ recorded on P. tritici at Beheira governorate.
\end{abstract}

Key words: Economic threshold and injury levels, Population density, Tetranychus urticae, Petrobia tritici, Wheat.

\section{INTRODUCTION}

Wheat is the most important cereal food crop for human and his animals in the world. Recently, the phytophagous mite species have been found infesting its plants in different wheat growing regions causing great damage. The two spotted spider mite, Tetranychus urticae Koch and the brown wheat mite, Petrobia tritici Kandeel, El-Naggar and Mohamed were recorded as serious mite species on wheat and other Gramineae hosts in many regions, i.e., rice and garlic plants are also infested with $P$. latens (Müller), Wang et al., (1994), Kride and Toit (1988), Noorbakhsh (1993), Prinsloo (2001) and Estal, et al. (1992). Also, this species harbored some weeds such as, Convolvulus spium, Faradji, (1995). In the same trend, Ibraheem et al.; (2007) studied the population densities of the two mite species and evaluated the response of three wheat verities to its infestation.

The use of economic thresholds as a basis for pest control decision is considered as a fundamental component of integrated pest management (Stern, et al., 1959). Proposed the concepts of an economic injury level (EIL) and economic threshold level (ETL) as rational comparison of the economic costs and benefits of pesticides use, EILs detected as the lowest number of pests cause economic damage, where the economic damage is the amount of damage that equal the control cost (Stern et al., 1959).

This study aimed to throw lights on assess the economic threshold, injury levels and population density of Tetranychus urticae and Petrobia tritici infestation as bases for decision making recommendation for the pest control programs in the field.

\section{MATERIALS AND METHODS}

\section{Assessment of economic damage threshold and economic injury levels:}

Two field experiments were carried out during 2016/2017 season in wheat fields at Hehia and Etay El-Barod districts (Sharkeia and Beheira governorates east and west nile delta ) to assess the Economic damage threshold and economic injury levels for the two dominant mite specie; the two spotted spider mite, Tetranychus urticae and the brown wheat mite, Petrobia tritici infest wheat cultivar Giza186, as Marking Plants (natural infestation technique).

\section{Marking plants experiment (Natural infestation technique):}

Sample of 40 plants were chosen randomly from $1 / 2$ fadan $\left(2100 \mathrm{~m}^{2}\right)$ cultivated with wheat (Giza 186 cultivar), from the beginning date of mite infestation and labeled, then left to natural infestation. The mite numbers/labeled plant were counted weekly in the field untill crop harvest. The seeds yield of each plant were collected, counted and weighed in gram per individual from genesis starting plants.

\section{Statistical analysis:}

Data were subjected to a certain scheme of statistical analysis, according to Hosny et al., (1972), Salem and Zaki (1985) and Ibrahim (1994 \& 2001) to calculate economic threshold and economic injury levels using: 


\section{Marking plants technique:}

The partial regression formula" c-multipliers" which has two independent variables $\left(\mathrm{x} 1{ }^{\text {and }} \mathrm{x} 2\right)$ were used, where the average number of mites per plant in the two peaks of population activity, the dependent variable (y) represented the yield per plant. The partial regression was used to show the variability in the yield that could be caused by infestation during the whole season. Standard error "SE", " $t$ " values, simple correlation ( $r$ ) and simple regression " $b$ " were calculated. The slope (b) of straight regression line was carried out to obtain the corrected values for the yield. The linear regression curve was obtained by transforming the (y) into logarithmic value using the following equation:

$$
y=e^{-(a+b x)}(i . e ., \log . Y= \pm b x)
$$

The chi-square analysis "rx2" was applied, the point at which the wheat at the upper part of the slope start to show a significant drop could be taken as a threshold level.

\section{Population fluctuation:}

Experiments were carried out at Hehia and Etay El-Barod districts during 2016/2017 season in wheat field cultivate at 20/ 11/2016. Weekly samples of 40 wheat leaves were randomly collected early in the morning from wheat fields in the two localities.

All collected samples sent to the laboratory. For mite examination. Temperature and relative humidity were also recorded.

All mite species were identified and the data were subjected to statistical analysis by using Costat Software program of little and Hills, (1975).

\section{RESULTS AND DISCUSSION}

\section{Assessment of economic threshold and economic injury levels:}

The Economic threshold of the two spotted spider mite and the brown wheat mite were studied using marking plants technique, where weekly counts of mites on 40 marked wheat plants were recorded and given in Tables (1:4) and in Figs. (1\&2). The mite fluctuation during the growing season showed that, Tetranychus urticae passed through 2 effective annual peaks on wheat plants at Beheira and Sharkia Governorates, subjected as X1 \& X2 ranged. Statistical analysis indicated that the considered factors (infestation through the two peaks of $T$. urticae) were responsible for 87.79 and $94.12 \%$ of the variability in the yield weight. The reduction in the yield at Sharkia and Beheira was affected by different manners from one peak to another. The infestation of the $26^{\text {th }}$ and $31^{\text {st }}$ of March (x2) showed relatively high effect on yield $(b=-1.84 \&-2.86)$ than in the first peak (x1) at $12^{\text {th }}$ and $3^{\text {rd }}$ March $(b=-1.39 \&-2.14)$ for the two localities respectively. Mathematic determination of the point at which the increase of mite species numbers through the two peaks of infestation $(\mathrm{X} 1 \& \mathrm{X} 2)$ caused a decrease in the weight of yield, chi-square analysis (rx2) was applied. Damage threshold was affected by the two infestation peaks. The mite number of the $1^{\text {st }}$ peak on $12^{\text {th }}$ and $3^{\text {rd }}$ March increased from 4.33 to $10.33 \& 4$ to 4.33 individuals/leaf that decreased yield from 115.05 to $100.18 \& 93.68$ to $84.51 \mathrm{gm}$. /plant. Also, for the $2^{\text {nd }}$ peak of $26^{\text {th }}$ and $31^{\text {st }}$ March, the mite numbers increased from 10 to 10.33 and from 4.33 to 5.00 individuals/leaf that decreased yield from 115.04 to 100.18 and from 56.56 to $55.46 \mathrm{gm}$. /plant at Sharkia and Beheira; respectively.

Obtained results agreed with those of Suekane et al.; (2012) who recorded that the number of seeds and seed weight were similarly affected by the twospotted spider mite, where the damage increased by increasing infestation.

In the same trend the ETLs of P.tritici on wheat plants in Sharkia and Beheira (as x $1 \& x 2$ peaks results), Table $(5,6,7 \& 8)$ and Figs; (3\&4) ranged 3.33-4.00 and 3.33 individuals. /leaf, while the economic injury level ranged 5-5.33 and 3.67-5.33 Individuals / leaf for the two localities, respectively.

Statistical analysis indicated that the considered factors (infestation through the two peaks considered of $P$. tritici population) were responsible for $79.68 \%$ and $96.02 \%$ of the variability in the yield weight. The wheat plants yield at Sharkia and Beheira were affected by different manner from one peak to another. The infestation of the $2^{\text {nd }}$ April and $31^{\text {st }}$ March (x2) showed relatively high effect on yield (b $=-3.17 \&-4.20)$ than in the first peak $(\mathrm{x} 1)$ at $5^{\text {th }}$ and $3^{\text {rd }}$ March $(b=-2.12 \&-2.08)$ for the two localities respectively.

Mathematic determination of the point at which the increase of mite species numbers through the two peaks of infestation (x1\&x2) caused a decrease in the weight of yield, chi-square analysis (rx2) was applied. The results showed that the damage threshold was affected by the two infestation peaks. The mite number during the $1^{\text {st }}$ peak of infestation $5^{\text {th }}$ and $3^{\text {rd }}$ March increased from 3.33 to 5.33 and from 3.33 to 5.33 individuals/leaf that decreased yield from 75.79 to 72.23 and from 62.85 to $60.99 \mathrm{gm}$. /plant. Also, for the $2^{\text {nd }}$ peaks of $2^{\text {nd }}$ April and $31^{\text {st }}$ March the mite numbers increased from 4.00 to 5.00 and from 3.33 to 3.67 individuals/leaf that decreased yield from 51.08 to 49.8 and from 60.99 to $59.07 \mathrm{gm}$. /plant at Sharkia and Beheira; respectively. 
Table (1): Yield-infestation relationship in 40 marked wheat plants under natural infestation with Tetranychus urticae during 2016/2017 season at Sharkia governorate

\begin{tabular}{|c|c|c|c|c|c|c|c|c|c|}
\hline \multirow[b]{2}{*}{$\begin{array}{l}\text { Plant } \\
\text { No. }\end{array}$} & \multicolumn{3}{|c|}{ OYAINPP } & \multicolumn{3}{|c|}{$1 \stackrel{\mathrm{ST}}{\text { peak }}$} & \multicolumn{3}{|c|}{$2^{\text {nd }}$ peak } \\
\hline & $\mathrm{x} 1$ & $\mathrm{x} 2$ & $\begin{array}{c}\text { Grain } \\
\text { weight } \\
\text { gm/plant }\end{array}$ & $\mathrm{x} 1$ & $\begin{array}{c}\text { Grain } \\
\text { weight } \\
\text { gm/plant }\end{array}$ & $\begin{array}{c}\text { Expected } \\
\text { grain weight } \\
\text { gm/plant }\end{array}$ & $\mathrm{x} 2$ & $\begin{array}{c}\text { Grain } \\
\text { weight } \\
\text { gm/plant }\end{array}$ & $\begin{array}{c}\text { Expected } \\
\text { grain weight } \\
\mathrm{gm} / \text { plant }\end{array}$ \\
\hline 1 & 4.33 & 61 & 26.0579 & 4.33 & 26.0579 & 74.11 & 10 & 26.0579 & 88.71 \\
\hline 2 & 28 & 25.33 & 76.4066 & 10.33 & 76.4066 & 65.74 & 13.3 & 76.4066 & 82.58 \\
\hline 3 & 33.33 & 45.67 & 33.4476 & 11.67 & 33.4476 & 63.87 & 15.3 & 33.4476 & 78.90 \\
\hline 4 & 44 & 25.33 & 13.1817 & 12.67 & 13.1817 & 62.48 & 19.3 & 13.1817 & 71.54 \\
\hline 5 & 67 & 40 & 25.6674 & 13.67 & 25.6674 & 61.08 & 20.6 & 25.6674 & 69.07 \\
\hline 6 & 51 & 31.33 & 17.0955 & 14.33 & 17.0955 & 60.16 & 22 & 17.0955 & 66.62 \\
\hline 7 & 40 & 23.33 & 18.2034 & 15 & 18.2034 & 59.23 & 23 & 18.2034 & 64.78 \\
\hline 8 & 60 & 43 & 10.74 & 15.67 & 10.74 & 58.29 & 23.3 & 10.74 & 64.17 \\
\hline 9 & 33.67 & 50.67 & 14.1225 & 16 & 14.1225 & 57.83 & 25.3 & 14.1225 & 60.49 \\
\hline 10 & 30.67 & 29 & 51.0848 & 16.33 & 51.0848 & 57.37 & 25.3 & 51.0848 & 60.49 \\
\hline 11 & 13.67 & 46.33 & 75.7872 & 16.67 & 75.7872 & 56.90 & 26 & 75.7872 & 59.26 \\
\hline 12 & 42 & 36 & 100.1836 & 19.33 & 100.1836 & 53.19 & 26.6 & 100.1836 & 58.03 \\
\hline 13 & 62.67 & 34.67 & 57.035 & 20 & 57.035 & 52.25 & 29 & 57.035 & 53.74 \\
\hline 14 & 43.33 & 60 & 28.0247 & 21 & 28.0247 & 50.86 & 31.3 & 28.0247 & 49.45 \\
\hline 15 & 67 & 35.33 & 40.5846 & 21.33 & 40.5846 & 50.40 & 32.3 & 40.5846 & 47.61 \\
\hline 16 & 20 & 52.67 & 36.2297 & 21.67 & 36.2297 & 49.92 & 33 & 36.2297 & 46.38 \\
\hline 17 & 19.33 & 45 & 41.0592 & 22.33 & 41.0592 & 49.00 & 33.3 & 41.0592 & 45.77 \\
\hline 18 & 21.33 & 65.67 & 9.0075 & 23 & 9.0075 & 48.07 & 34.6 & 9.0075 & 43.30 \\
\hline 19 & 25.33 & 38 & 19.9494 & 23.33 & 19.9494 & 47.61 & 35.3 & 19.9494 & 42.09 \\
\hline 20 & 21 & 54 & 22.136 & 24 & 22.136 & 46.67 & 36 & 22.136 & 40.85 \\
\hline 21 & 16.67 & 26 & 49.7952 & 25.33 & 49.7952 & 44.82 & 38 & 49.7952 & 37.17 \\
\hline 22 & 16.33 & 44.33 & 58.2335 & 28 & 58.2335 & 41.09 & 40 & 58.2335 & 33.49 \\
\hline 23 & 15.67 & 15.33 & 12.944 & 30 & 12.944 & 38.30 & 41.6 & 12.944 & 30.42 \\
\hline 24 & 35 & 53.67 & 16.2384 & 30.67 & 16.2384 & 37.37 & 42 & 16.2384 & 29.81 \\
\hline 25 & 23.33 & 26.67 & 53.187 & 32.33 & 53.187 & 35.05 & 43 & 53.187 & 27.97 \\
\hline 26 & 23 & 22 & 85.0626 & 33 & 85.0626 & 34.12 & 43.3 & 85.0626 & 27.36 \\
\hline 27 & 32.33 & 50 & 68.391 & 33.33 & 68.391 & 33.66 & 44 & 68.391 & 26.13 \\
\hline 28 & 16 & 20.67 & 32.2572 & 33.67 & 32.2572 & 33.18 & 44.3 & 32.2572 & 25.52 \\
\hline 29 & 10.33 & 13.33 & 115.0437 & 35 & 115.0437 & 31.33 & 45 & 115.0437 & 24.29 \\
\hline 30 & 11.67 & 10 & 81.058 & 40 & 81.058 & 24.35 & 45.6 & 81.058 & 23.06 \\
\hline 31 & 30 & 41.67 & 11.94 & 42 & 11.94 & 21.56 & 46.3 & 11.94 & 21.84 \\
\hline 32 & 33 & 33.33 & 20.1024 & 43 & 20.1024 & 20.17 & 50 & 20.1024 & 15.09 \\
\hline 33 & 12.67 & 33 & 23.0355 & 43.33 & 23.0355 & 19.71 & 50.6 & 23.0355 & 13.85 \\
\hline 34 & 14.33 & 43.33 & 72.23463 & 44 & 72.23463 & 18.77 & 52.6 & 72.23463 & 10.17 \\
\hline 35 & 63.33 & 19.33 & 10.35 & 51 & 10.35 & 9.01 & 53.6 & 10.35 & 8.33 \\
\hline 36 & 21.67 & 62.67 & 8.368 & 60 & 8.368 & 2.15 & 54 & 8.368 & 7.72 \\
\hline 37 & 64.33 & 23 & 5.6871 & 62.67 & 5.6871 & 4.95 & 60 & 5.6871 & 8.71 \\
\hline 38 & 24 & 42 & 29.3525 & 63.33 & 29.3525 & 4.16 & 61 & 29.3525 & 7.07 \\
\hline 39 & 15 & 32.33 & 24.373 & 64.33 & 24.373 & 2.96 & 62.6 & 24.373 & 4.96 \\
\hline 40 & 22.33 & 44 & 31.9188 & 67 & 31.9188 & 1.76 & 65.6 & 31.9188 & 2.04 \\
\hline
\end{tabular}


Table (2): Yield-infestation relationship in 40 marked wheat plants under natural infestation with Tetranychus urticae during 2016/2017 season at Beheira governorate

\begin{tabular}{|c|c|c|c|c|c|c|c|c|c|}
\hline \multirow[b]{2}{*}{$\begin{array}{l}\text { Plant } \\
\text { No. }\end{array}$} & \multicolumn{3}{|c|}{ OYAINPP } & \multicolumn{3}{|c|}{$1^{\text {st }}$ peak } & \multicolumn{3}{|c|}{$2^{\text {nd }}$ peak } \\
\hline & $\mathrm{x} 1$ & $\mathrm{x} 2$ & $\begin{array}{c}\text { Grain } \\
\text { weight } \\
\text { gm/plant }\end{array}$ & $\mathrm{x} 1$ & $\begin{array}{c}\text { Grain weight } \\
\text { gm/plant }\end{array}$ & $\begin{array}{l}\text { Expected } \\
\text { grain } \\
\text { weight } \\
\text { gm/plant }\end{array}$ & $\mathrm{x} 2$ & $\begin{array}{c}\text { Grain } \\
\text { weight } \\
\text { gm/plant }\end{array}$ & $\begin{array}{l}\text { Expected } \\
\text { grain } \\
\text { weight } \\
\text { gm/plant }\end{array}$ \\
\hline 1 & 26.33 & 20 & 69.037 & 26.33 & 69.037 & 70.65 & 20 & 69.037 & 69.60 \\
\hline 2 & 5.33 & 1.67 & 5.0094 & 5.33 & 5.0094 & 69.94 & 1.67 & 5.0094 & 68.63 \\
\hline 3 & 6 & 2 & 22.8 & 6 & 22.8 & 68.51 & 2 & 22.8 & 66.74 \\
\hline 4 & 10.67 & 0.67 & 24.729 & 10.67 & 24.729 & 67.80 & 0.67 & 24.729 & 65.77 \\
\hline 5 & 11.33 & 4 & 41.912 & 11.33 & 41.912 & 66.36 & 4 & 41.912 & 64.82 \\
\hline 6 & 17 & 3.33 & 39.949 & 17 & 39.949 & 62.78 & 3.33 & 39.949 & 63.88 \\
\hline 7 & 4.33 & 7.67 & 29.24 & 4.33 & 29.24 & 57.79 & 7.67 & 29.24 & 62.90 \\
\hline 8 & 13.33 & 5 & 26.147 & 13.33 & 26.147 & 56.35 & 5 & 26.147 & 61.02 \\
\hline 9 & 19.67 & 13.67 & 7.647 & 19.67 & 7.647 & 54.94 & 13.67 & 7.647 & 59.10 \\
\hline 10 & 13.67 & 5.33 & 36.16 & 13.67 & 36.16 & 54.21 & 5.33 & 36.16 & 58.15 \\
\hline 11 & 43.33 & 24 & 32.135 & 43.33 & 32.135 & 50.65 & 24 & 32.135 & 56.24 \\
\hline 12 & 17.67 & 8 & 65.0685 & 17.67 & 65.0685 & 49.92 & 8 & 65.0685 & 55.29 \\
\hline 13 & 11.67 & 4.33 & 17.2296 & 11.67 & 17.2296 & 47.07 & 4.33 & 17.2296 & 54.32 \\
\hline 14 & 4 & 6 & 10.7406 & 4 & 10.7406 & 46.36 & 6 & 10.7406 & 53.38 \\
\hline 15 & 15.33 & 20.67 & 56.56 & 15.33 & 56.56 & 44.93 & 20.67 & 56.56 & 49.57 \\
\hline 16 & 30 & 15 & 34.946 & 30 & 34.946 & 42.78 & 15 & 34.946 & 48.60 \\
\hline 17 & 22 & 0.33 & 25.7862 & 22 & 25.7862 & 41.35 & 0.33 & 25.7862 & 47.65 \\
\hline 18 & 7.67 & 2.33 & 1.7989 & 7.67 & 1.7989 & 40.64 & 2.33 & 1.7989 & 44.79 \\
\hline 19 & 23.33 & 18 & 50.7732 & 23.33 & 50.7732 & 39.93 & 18 & 50.7732 & 41.93 \\
\hline 20 & 24 & 18.33 & 59.0715 & 24 & 59.0715 & 38.49 & 18.33 & 59.0715 & 38.12 \\
\hline 21 & 5 & 1.33 & 60.9915 & 5 & 60.9915 & 37.06 & 1.33 & 60.9915 & 31.42 \\
\hline 22 & 22.33 & 16.67 & 46.3545 & 22.33 & 46.3545 & 36.35 & 16.67 & 46.3545 & 29.54 \\
\hline 23 & 30.33 & 21 & 9.7902 & 30.33 & 9.7902 & 32.06 & 21 & 9.7902 & 27.62 \\
\hline 24 & 25 & 19 & 33.942 & 25 & 33.942 & 31.36 & 19 & 33.942 & 22.84 \\
\hline 25 & 15 & 5.67 & 13.6712 & 15 & 13.6712 & 30.63 & 5.67 & 13.6712 & 21.90 \\
\hline 26 & 23 & 17.67 & 58.7485 & 23 & 58.7485 & 29.92 & 17.67 & 58.7485 & 19.98 \\
\hline 27 & 32 & 21.67 & 49.5205 & 32 & 49.5205 & 29.21 & 21.67 & 49.5205 & 19.03 \\
\hline 28 & 22.67 & 17 & 12.9788 & 22.67 & 12.9788 & 27.78 & 17 & 12.9788 & 18.09 \\
\hline 29 & 20 & 14.33 & 19.5435 & 20 & 19.5435 & 25.63 & 14.33 & 19.5435 & 16.17 \\
\hline 30 & 18 & 9 & 84.5064 & 18 & 84.5064 & 24.93 & 9 & 84.5064 & 14.25 \\
\hline 31 & 10 & 2.67 & 55.467 & 10 & 55.467 & 22.78 & 2.67 & 55.467 & 13.31 \\
\hline 32 & 35 & 22.33 & 93.6786 & 35 & 93.6786 & 14.91 & 22.33 & 93.6786 & 11.39 \\
\hline 33 & 25.33 & 19.67 & 18.0282 & 25.33 & 18.0282 & 14.21 & 19.67 & 18.0282 & 10.45 \\
\hline 34 & 10.33 & 10 & 63.2695 & 10.33 & 63.2695 & 10.63 & 10 & 63.2695 & 8.53 \\
\hline 35 & 40 & 23.33 & 11.9402 & 40 & 11.9402 & 7.78 & 23.33 & 11.9402 & 7.59 \\
\hline 36 & 33.33 & 22 & 21.9351 & 33.33 & 21.9351 & 4.20 & 22 & 21.9351 & 6.64 \\
\hline 37 & 16 & 7.33 & 3.3651 & 16 & 3.3651 & 3.49 & 7.33 & 3.3651 & 5.67 \\
\hline 38 & 19 & 11.33 & 2.0882 & 19 & 2.0882 & 3.22 & 11.33 & 2.0882 & 3.78 \\
\hline 39 & 35.33 & 22.67 & 62.8465 & 35.33 & 62.8465 & 3.62 & 22.67 & 62.8465 & 2.81 \\
\hline 40 & 42 & 23.67 & 43.9488 & 42 & 43.9488 & 5.56 & 23.67 & 43.9488 & 1.86 \\
\hline
\end{tabular}


Table (3): Statistical analysis Simple correlation(r) and Partial regression (b1\&b2) for the relationship between Tetranychus urticae (Sayed) peaks count and the yield of 40 marked wheat plants (weight of grain) in Sharkia during 2017season

\begin{tabular}{cccccccc}
\hline \multirow{2}{*}{ Variables } & \multicolumn{2}{c}{$\begin{array}{c}\text { Simple } \\
\text { correlation(r) }\end{array}$} & \multicolumn{3}{c}{ Partial regression } & Explained variance \\
\cline { 2 - 8 } & $\mathrm{R}$ & $\mathrm{p}$ & $\mathrm{b}$ & $\mathrm{S} . \mathrm{E}$ & $\mathrm{t}$ & probability & EV\% \\
\hline mite no./ plant (x1) & -0.83 & $\leq 0.01$ & -1.39 & 0.15 & -9.29 & $\leq 0.01$ & 69.47 \\
\hline mite no./ plant (x2) & -0.94 & $\leq 0.01$ & -1.84 & 0.11 & -16.52 & $\leq 0.01$ & 87.79
\end{tabular}

$\mathrm{r}=$ correlation coefficient, $\mathrm{b}=$ Partial regression values, $\mathrm{x} 1=1^{\text {st }}$ peak and $\mathrm{x} 2=2^{\text {nd }}$ peak

Table (4) Statistical analysis Simple correlation(r) and Partial regression (b1\&b2) for the relationship between Tetranychus urticae (Sayed) peaks count and the yield of 40 marked wheat plants (weight of grain) in Beheira during 2017season

\begin{tabular}{cccccccc}
\hline \multirow{2}{*}{ Variables } & \multicolumn{3}{c}{ Simple correlation(r) } & \multicolumn{3}{c}{ Partial regression } & Explained variance \\
\cline { 2 - 8 } & $\mathrm{R}$ & $\mathrm{p}$ & $\mathrm{b}$ & $\mathrm{S} . \mathrm{E}$ & $\mathrm{t}$ & probability & EV\% \\
\hline mite no./plant (x1) & -0.95 & $\leq 0.01$ & -2.14 & 0.11 & -19.57 & $\leq 0.01$ & 90.98 \\
\hline mite no./plant (x2) & -0.97 & $\leq 0.01$ & -2.86 & 0.12 & -24.66 & $\leq 0.01$ & 94.12
\end{tabular}

$\mathrm{r}=$ correlation coefficient, $\mathrm{b}=$ Partial regression values, $\mathrm{x} 1=1^{\text {st }}$ peak and $\mathrm{x} 2=2^{\text {nd }}$ peak.

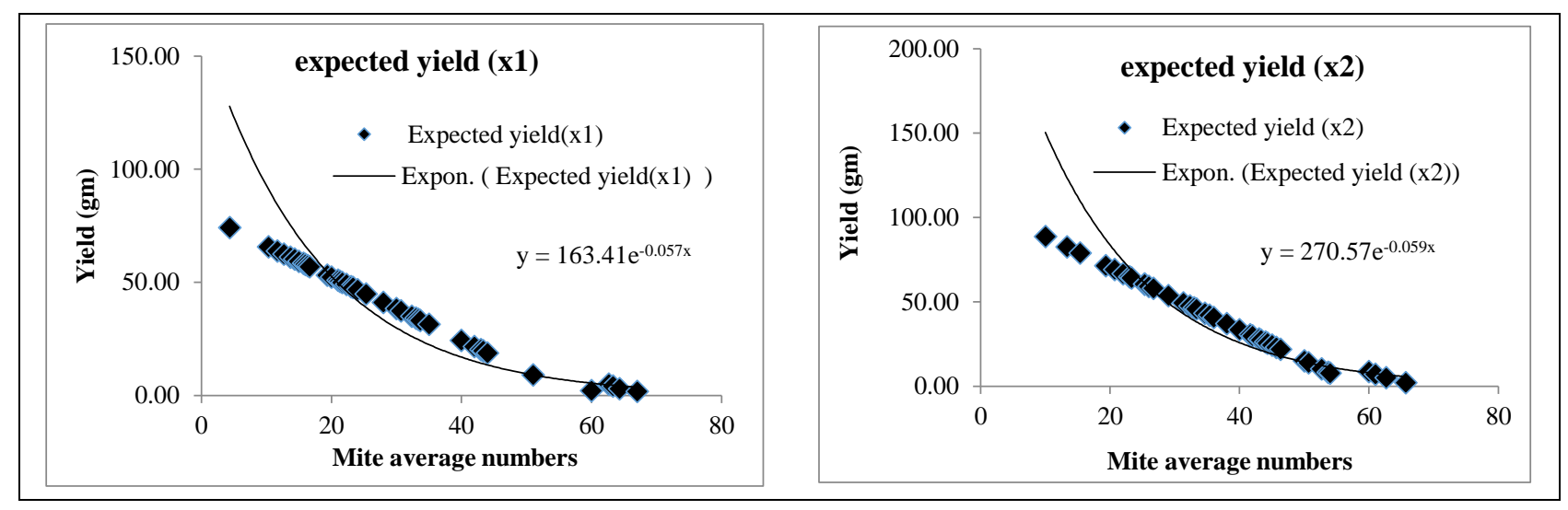

Fig (1): The corrected average change in the wheat plants yield (y) per unit change in Tetranychus urticae infestation at the first (x1) and the second (x2) annual peaks at Sharkia Governorate during 2016/2017 season.

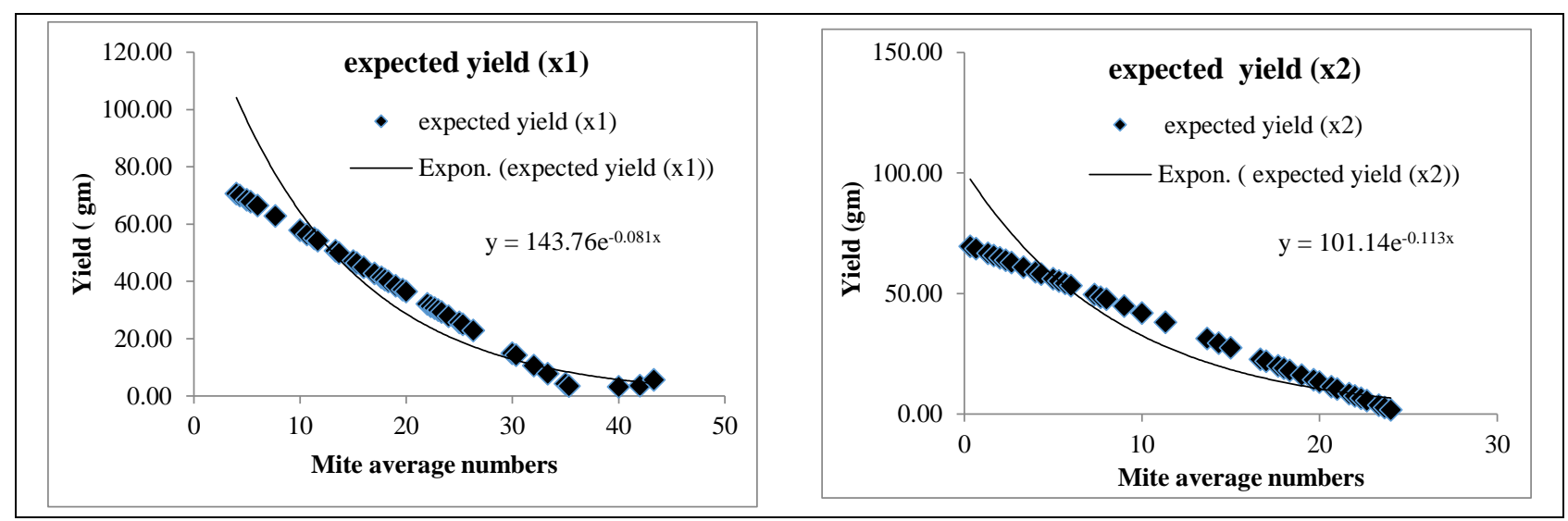

Fig (2): The corrected average change in the wheat plants yield (y)per unit change in Tetranychus urticae infestation at the first (x1) and the second (x2) annual peaks at Behira Governorate during 2016/2017 season. 
Table (5): Yield-infestation relationship in 40 marked wheat plants under natural infestation with Petrobia tritici during 2016/2017 season at Sharkia Governorate

\begin{tabular}{|c|c|c|c|c|c|c|c|c|c|}
\hline \multirow{2}{*}{$\begin{array}{c}\text { Plant } \\
\text { No. }\end{array}$} & \multicolumn{3}{|c|}{ OYAINPP } & \multicolumn{3}{|c|}{$1 \stackrel{\mathrm{ST}}{\text { peak }}$} & \multicolumn{3}{|c|}{$2^{\text {nd }}$ peak } \\
\hline & $\mathrm{x} 1$ & $\mathrm{x} 2$ & $\begin{array}{c}\text { Grain } \\
\text { weight } \\
\text { gm/plant }\end{array}$ & $\mathrm{x} 1$ & $\begin{array}{c}\text { Grain } \\
\text { weight } \\
\text { gm/plant }\end{array}$ & $\begin{array}{l}\text { Expected } \\
\text { grain weight } \\
\text { gm/plant }\end{array}$ & $\mathrm{x} 2$ & $\begin{array}{c}\text { Grain } \\
\text { weight } \\
\text { gm/plant }\end{array}$ & $\begin{array}{c}\text { Expected grain } \\
\text { weight } \\
\text { gm/plant }\end{array}$ \\
\hline 1 & 2.33 & 3.33 & 26.0579 & 2.33 & 26.0579 & 70.44 & 3.33 & 26.0579 & 71.86 \\
\hline 2 & 21 & 2.67 & 76.4066 & 21 & 76.4066 & 69.04 & 2.67 & 76.4066 & 70.78 \\
\hline 3 & 19 & 6 & 33.4476 & 19 & 33.4476 & 68.32 & 6 & 33.4476 & 69.74 \\
\hline 4 & 11.33 & & 13.1817 & 11.33 & 13.1817 & 67.62 & & 13.1817 & 68.69 \\
\hline 5 & 5.67 & 2 & 25.6674 & 5.67 & 25.6674 & 66.92 & 2 & 25.6674 & 67.62 \\
\hline 6 & 9 & 1 & 17.0955 & 9 & 17.0955 & 64.79 & 1 & 17.0955 & 66.57 \\
\hline 7 & 14.33 & 1.67 & 18.2034 & 14.33 & 18.2034 & 60.55 & 1.67 & 18.2034 & 65.52 \\
\hline 8 & 18 & 8 & 10.74 & 18 & 10.74 & 59.83 & 8 & 10.74 & 64.45 \\
\hline 9 & 38 & 7.33 & 14.1225 & 38 & 14.1225 & 59.13 & 7.33 & 14.1225 & 63.40 \\
\hline 10 & 22 & 18.67 & 51.0848 & 22 & 51.0848 & 56.30 & 18.67 & 51.0848 & 62.36 \\
\hline 11 & 29.33 & 0.67 & 75.7872 & 29.33 & 75.7872 & 54.88 & 0.67 & 75.7872 & 61.28 \\
\hline 12 & 19.67 & 5 & 100.1836 & 19.67 & 100.1836 & 52.76 & 5 & 100.1836 & 60.23 \\
\hline 13 & 32 & 8.67 & 57.035 & 32 & 57.035 & 51.33 & 8.67 & 57.035 & 57.07 \\
\hline 14 & 17 & 20 & 28.0247 & 17 & 28.0247 & 50.63 & 20 & 28.0247 & 56.02 \\
\hline 15 & 15 & 11.33 & 40.5846 & 15 & 40.5846 & 49.93 & 11.33 & 40.5846 & 54.94 \\
\hline 16 & 21.67 & 12 & 36.2297 & 21.67 & 36.2297 & 47.81 & 12 & 36.2297 & 53.90 \\
\hline 17 & 10.33 & 15.67 & 41.0592 & 10.33 & 41.0592 & 47.09 & 15.67 & 41.0592 & 49.69 \\
\hline 18 & 8 & 9.33 & 9.0075 & 8 & 9.0075 & 46.38 & 9.33 & 9.0075 & 47.56 \\
\hline 19 & 0.67 & 0.33 & 19.9494 & 0.67 & 19.9494 & 43.56 & 0.33 & 19.9494 & 45.44 \\
\hline 20 & 1.33 & 10 & 22.136 & 1.33 & 22.136 & 42.84 & 10 & 22.136 & 43.35 \\
\hline 21 & 2 & 3 & 49.7952 & 2 & 49.7952 & 42.14 & 3 & 49.7952 & 41.23 \\
\hline 22 & 7.33 & 14.67 & 58.2335 & 7.33 & 58.2335 & 41.44 & 14.67 & 58.2335 & 37.01 \\
\hline 23 & 47 & 1.33 & 12.944 & 47 & 12.944 & 40.01 & 1.33 & 12.944 & 34.89 \\
\hline 24 & 14 & 16.33 & 16.2384 & 14 & 16.2384 & 37.89 & 16.33 & 16.2384 & 30.68 \\
\hline 25 & 11.67 & 12.33 & 53.187 & 11.67 & 53.187 & 35.77 & 12.33 & 53.187 & 26.43 \\
\hline 26 & 30 & 4 & 85.0626 & 30 & 85.0626 & 33.64 & 4 & 85.0626 & 25.39 \\
\hline 27 & 1.67 & 15 & 68.391 & 1.67 & 68.391 & 31.52 & 15 & 68.391 & 23.27 \\
\hline 28 & 29 & 5.67 & 32.2572 & 29 & 32.2572 & 30.10 & 5.67 & 32.2572 & 21.17 \\
\hline 29 & 13.33 & 19.33 & 115.0437 & 13.33 & 115.0437 & 27.27 & 19.33 & 115.0437 & 18.01 \\
\hline 30 & 16 & 17.33 & 81.058 & 16 & 81.058 & 25.85 & 17.33 & 81.058 & 15.88 \\
\hline 31 & 35 & 20.33 & 11.94 & 35 & 11.94 & 25.15 & 20.33 & 11.94 & 13.76 \\
\hline 32 & 3.33 & 21 & 20.1024 & 3.33 & 20.1024 & 24.45 & 21 & 20.1024 & 11.67 \\
\hline 33 & 13.67 & 22.33 & 23.0355 & 13.67 & 23.0355 & 10.29 & 22.33 & 23.0355 & 9.55 \\
\hline 34 & 12 & 21.67 & 72.23463 & 12 & 72.23463 & 9.59 & 21.67 & 72.23463 & 8.50 \\
\hline 35 & 22.33 & 23 & 10.35 & 22.33 & 10.35 & 8.16 & 23 & 10.35 & 6.38 \\
\hline 36 & 6 & 5.33 & 8.368 & 6 & 8.368 & 7.46 & 5.33 & 8.368 & 4.26 \\
\hline 37 & 5.33 & 18 & 5.6871 & 5.33 & 5.6871 & 3.92 & 18 & 5.6871 & 2.17 \\
\hline 38 & 9.67 & 23.67 & 29.3525 & 9.67 & 29.3525 & 1.87 & 23.67 & 29.3525 & 0.05 \\
\hline 39 & 30.33 & 3.67 & 24.373 & 30.33 & 24.373 & 3.47 & 3.67 & 24.373 & 1.90 \\
\hline 40 & 10 & 24 & 31.9188 & 10 & 31.9188 & 1.37 & 24 & 31.9188 & 1.39 \\
\hline
\end{tabular}


Table (6): Yield-infestation relationship in 40 marked wheat plants under natural infestation with Petrobia tritici during 2016/2017 season at Beheira governorate

\begin{tabular}{|c|c|c|c|c|c|c|c|c|c|}
\hline \multirow[t]{2}{*}{$\begin{array}{l}\text { Plant } \\
\text { NO. }\end{array}$} & \multicolumn{3}{|c|}{ OYAINPP } & \multicolumn{3}{|c|}{$1 \stackrel{\text { ST }}{\text { peak }}$} & \multicolumn{3}{|c|}{$2^{\text {nd }}$ peak } \\
\hline & $\mathrm{x} 1$ & $\mathrm{x} 2$ & $\begin{array}{c}\text { Grain } \\
\text { weight } \\
\text { gm/plant }\end{array}$ & $\mathrm{x} 1$ & $\begin{array}{c}\text { Grain } \\
\text { weight } \\
\text { gm/plant }\end{array}$ & $\begin{array}{l}\text { Expected } \\
\text { grain weight } \\
\text { gm/plant }\end{array}$ & $\mathrm{x} 2$ & $\begin{array}{c}\text { Grain } \\
\text { weight } \\
\text { gm/plant }\end{array}$ & $\begin{array}{l}\text { Expected } \\
\text { grain weight } \\
\text { gm/plant }\end{array}$ \\
\hline 1 & 2.33 & 13 & 69.037 & 2.33 & 69.037 & 66.51 & 13 & 69.037 & 73.67 \\
\hline 2 & 21.67 & 2 & 5.0094 & 21.67 & 5.0094 & 65.14 & 2 & 5.0094 & 70.90 \\
\hline 3 & 11.67 & 6 & 22.8 & 11.67 & 22.8 & 64.43 & 6 & 22.8 & 69.47 \\
\hline 4 & 2 & 8 & 24.729 & 2 & 24.729 & 63.74 & 8 & 24.729 & 68.08 \\
\hline 5 & 9 & 1.33 & 41.912 & 9 & 41.912 & 63.06 & 1.33 & 41.912 & 66.70 \\
\hline 6 & 14.67 & 10 & 39.949 & 14.67 & 39.949 & 60.97 & 10 & 39.949 & 65.27 \\
\hline 7 & 18 & 2.33 & 29.24 & 18 & 29.24 & 56.81 & 2.33 & 29.24 & 62.49 \\
\hline 8 & 7.67 & 12.33 & 26.147 & 7.67 & 26.147 & 56.10 & 12.33 & 26.147 & 61.07 \\
\hline 9 & 22 & 4 & 7.647 & 22 & 7.647 & 55.42 & 4 & 7.647 & 59.68 \\
\hline 10 & 29 & 0.67 & 36.16 & 29 & 36.16 & 52.65 & 0.67 & 36.16 & 55.48 \\
\hline 11 & 19.33 & 5 & 32.135 & 19.33 & 32.135 & 51.94 & 5 & 32.135 & 54.09 \\
\hline 12 & 32.67 & 17.33 & 65.0685 & 32.67 & 65.0685 & 51.25 & 17.33 & 65.0685 & 52.66 \\
\hline 13 & 7.33 & 12.67 & 17.2296 & 7.33 & 17.2296 & 49.17 & 12.67 & 17.2296 & 51.27 \\
\hline 14 & 17 & 3.67 & 10.7406 & 17 & 10.7406 & 47.09 & 3.67 & 10.7406 & 48.46 \\
\hline 15 & 15.67 & 18.33 & 56.56 & 15.67 & 56.56 & 46.40 & 18.33 & 56.56 & 45.69 \\
\hline 16 & 10.33 & 11.67 & 34.946 & 10.33 & 34.946 & 44.32 & 11.67 & 34.946 & 44.26 \\
\hline 17 & 8 & 1.67 & 25.7862 & 8 & 25.7862 & 43.61 & 1.67 & 25.7862 & 42.87 \\
\hline 18 & 0.67 & 3.33 & 1.7989 & 0.67 & 1.7989 & 42.92 & 3.33 & 1.7989 & 40.06 \\
\hline 19 & 1.33 & 10.33 & 50.7732 & 1.33 & 50.7732 & 40.15 & 10.33 & 50.7732 & 38.67 \\
\hline 20 & 22.67 & 7.67 & 59.0715 & 22.67 & 59.0715 & 39.45 & 7.67 & 59.0715 & 35.85 \\
\hline 21 & 47 & 13.67 & 60.9915 & 47 & 60.9915 & 37.36 & 13.67 & 60.9915 & 34.47 \\
\hline 22 & 24.67 & 5.33 & 46.3545 & 24.67 & 46.3545 & 36.68 & 5.33 & 46.3545 & 33.08 \\
\hline 23 & 30 & 17.67 & 9.7902 & 30 & 9.7902 & 35.28 & 17.67 & 9.7902 & 31.65 \\
\hline 24 & 1.67 & 19 & 33.942 & 1.67 & 33.942 & 34.60 & 19 & 33.942 & 30.26 \\
\hline 25 & 13.33 & 14 & 13.6712 & 13.33 & 13.6712 & 32.51 & 14 & 13.6712 & 27.45 \\
\hline 26 & 16 & 15.67 & 58.7485 & 16 & 58.7485 & 30.43 & 15.67 & 58.7485 & 24.67 \\
\hline 27 & 35.33 & 6.67 & 49.5205 & 35.33 & 49.5205 & 27.66 & 6.67 & 49.5205 & 23.25 \\
\hline 28 & 3.33 & 9 & 12.9788 & 3.33 & 12.9788 & 22.79 & 9 & 12.9788 & 21.86 \\
\hline 29 & 13.67 & 11 & 19.5435 & 13.67 & 19.5435 & 22.10 & 11 & 19.5435 & 19.04 \\
\hline 30 & 12 & 18.67 & 84.5064 & 12 & 84.5064 & 20.71 & 18.67 & 84.5064 & 17.66 \\
\hline 31 & 25 & 5.67 & 55.467 & 25 & 55.467 & 20.02 & 5.67 & 55.467 & 14.84 \\
\hline 32 & 5.33 & 9.67 & 93.6786 & 5.33 & 93.6786 & 18.63 & 9.67 & 93.6786 & 12.07 \\
\hline 33 & 23.67 & 8.67 & 18.0282 & 23.67 & 18.0282 & 16.54 & 8.67 & 18.0282 & 10.64 \\
\hline 34 & 15 & 16 & 63.2695 & 15 & 63.2695 & 15.86 & 16 & 63.2695 & 9.25 \\
\hline 35 & 23 & 15.33 & 11.9402 & 23 & 11.9402 & 13.78 & 15.33 & 11.9402 & 6.44 \\
\hline 36 & 6 & 2.67 & 21.9351 & 6 & 21.9351 & 7.53 & 2.67 & 21.9351 & 3.66 \\
\hline 37 & 5.67 & 10.33 & 3.3651 & 5.67 & 3.3651 & 5.45 & 10.33 & 3.3651 & 2.23 \\
\hline 38 & 26 & 14.67 & 2.0882 & 26 & 2.0882 & 5.83 & 14.67 & 2.0882 & 3.17 \\
\hline 39 & 11.33 & 7.33 & 62.8465 & 11.33 & 62.8465 & 4.31 & 7.33 & 62.8465 & 1.81 \\
\hline 40 & 10 & 16.67 & 43.9488 & 10 & 43.9488 & 6.81 & 16.67 & 43.9488 & 0.87 \\
\hline
\end{tabular}


Table (7): Statistical analysis Simple correlation(r) and Partial regression (b1\&b2)for the relationship between Petrobia tritici Kandeel, El-Naggar and Mohamed peaks count and the yield of 40 marked wheat plants (weight of grain) Sharkia gov. during 2017season

\begin{tabular}{cccccccc}
\hline \multirow{2}{*}{ Variables } & \multicolumn{2}{c}{ Simple correlation(r) } & \multicolumn{3}{c}{ Partial regression } & \multicolumn{2}{c}{ Explained variance } \\
\cline { 2 - 7 } & $\mathrm{R}$ & $\mathrm{p}$ & $\mathrm{b}$ & $\mathrm{S} . \mathrm{E}$ & $\mathrm{t}$ & probability & EV\% \\
\hline Mite no./ plant $(\mathrm{x} 1)$ & -0.85 & $\leq 0.01$ & -2.12 & 0.21 & -10.07 & $\leq 0.01$ & 72.77 \\
\hline Mite no./ plant (x2) & -0.89 & $\leq 0.01$ & -3.17 & 0.26 & -12.21 & $\leq 0.01$ & 79.68 \\
\hline & $\mathrm{r}=$ correlation coefficient, $\mathrm{b}=$ Partial regression values, $\mathrm{x} 1=1^{\text {st }}$ peak and $\mathrm{x} 2=2^{\text {nd }}$ peak
\end{tabular}

Table (8): Statistical analysis Simple correlation(r) and Partial regression (b1\&b2)for the relationship between Petrobia tritici Kandeel, El-Naggar and Mohamed peaks count and the yield of 40 marked wheat plants (weight of grain) Beheira gov. during 2017season

\begin{tabular}{cccccccc}
\hline \multirow{2}{*}{ Variables } & \multicolumn{2}{c}{ Simple correlation(r) } & \multicolumn{2}{c}{ Partial regression } & \multicolumn{2}{c}{ Explained variance } \\
\cline { 2 - 7 } & $\mathrm{R}$ & $\mathrm{p}$ & $\mathrm{b}$ & $\mathrm{S} . \mathrm{E}$ & $\mathrm{t}$ & probability & EV\% \\
\hline Mite no./ plant (x1) & -0.93 & $\leq 0.01$ & -2.08 & 0.13 & -15.54 & $\leq 0.01$ & 86.41 \\
\hline Mite no./ plant (x2) & -0.98 & $\leq 0.01$ & -4.20 & 0.14 & -30.26 & $\leq 0.01$ & 96.02 \\
\hline
\end{tabular}
$\mathrm{r}=$ correlation coefficient, $\mathrm{b}=$ Partial regression values, $\mathrm{x} 1=1^{\text {st }}$ peak and $\mathrm{x} 2=2^{\text {nd }}$ peak

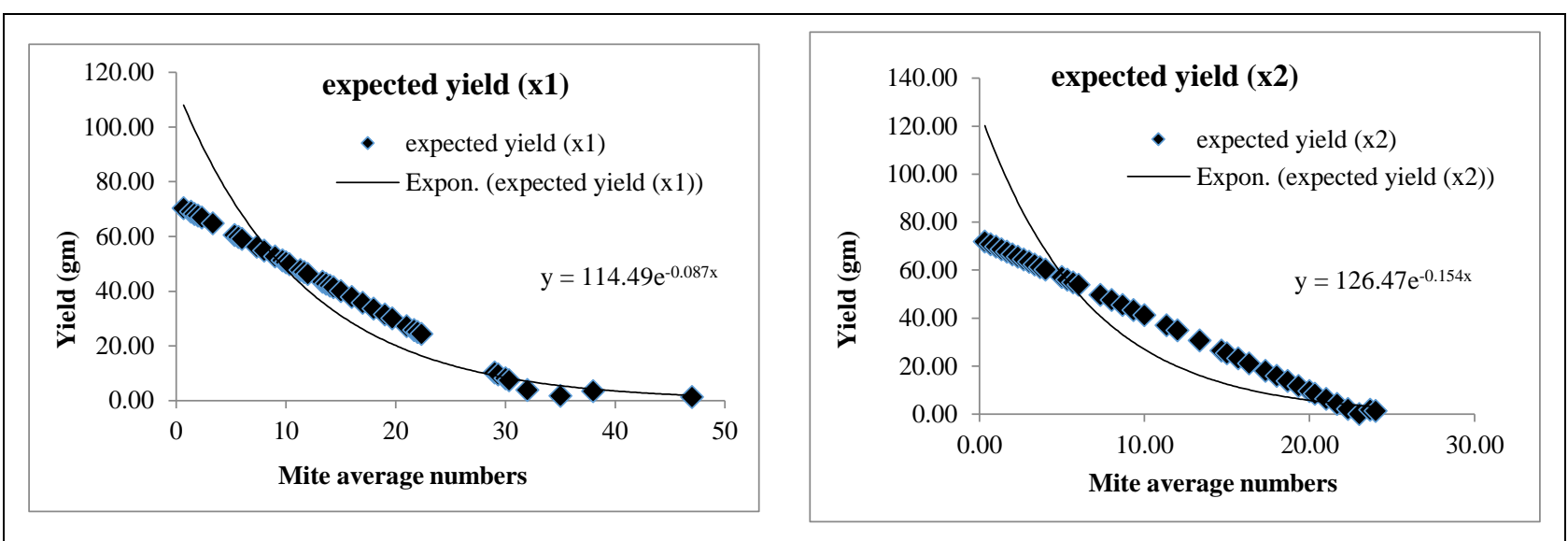

Fig (3): The corrected average change in the wheat plants yield (y)per unit change in Petrobia tritici infestation at the first (x1) and the second (x2) annual peaks at Sharkia Governorate during 2016/2017 season.

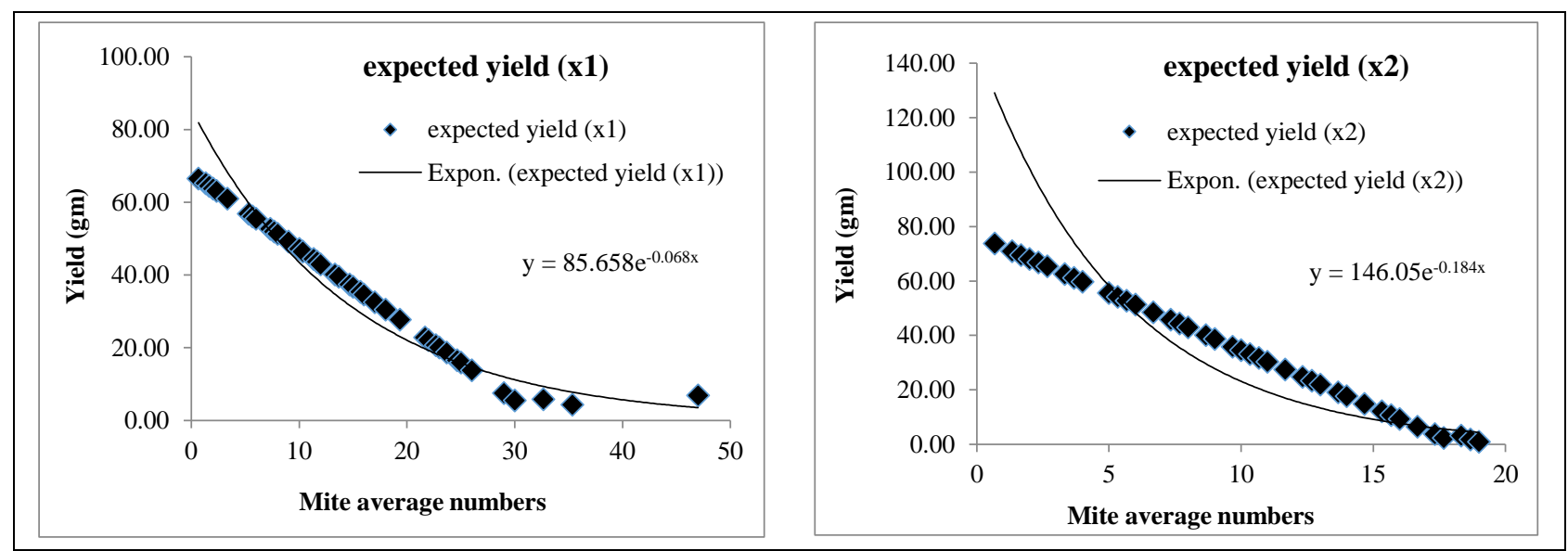

Fig (4): The corrected average change in the wheat plants yield (y) per unit change in Petrobia tritici infestation at the first (x1) and the second (x2) annual peaks at Behira Governorate during 2016/2017 season.

1. Population density of T. urticae and P. tritici infested wheat plants:

Tables (9 and10) showed two peaks for each species at the two localities, and the highest peaks of 37.47 and 20.36 individuals /leaf, were recorded on $26^{\text {th }}$ and $3^{\text {rd }}$ March for T.urticae at Sharkia and Beheira respectively; where the highest peaks of
15.88 and 15.53 individuals /leaf, were on $5^{\text {th }}$ and $3^{\text {rd }}$ March for P.tritici at the two governorates, respectively.

The results of statistical analysis detected that positive insignificant correlation was observed between the population of the two-mite species and 
temperature while it was negative and insignificant with relative humidity. The relatively high effect as partial regression (E.V. \%) for tested climatic factors of $5.18 \%$ was recorded for temperature on P.tritici at Sharkia governorate, while the relatively high multiple correlation for the two tested climatic factors together was $17.07 \%$ recorded on $P$. tritici at Beheira governorate.

\section{ACKNOWLEDGMENTS}

Many thanks to Prof. Dr. Mohamed Mohamed Ibrahim for his sincere guidance and the unlimited help he gave during the experimental work and during the preparation and correction of the manuscript.

\section{REFERENCES}

Estal, P. Del; M. Arroyo; E. Vinuela and F. Budia (1985): Pest mites of garlic in Spain. Anuales del Instituto Nacional de Investigaoins Agrarias Serie Agricota 28(sp No) 131-145.

Faradji, F. (1995): Report of Tetranychina harti (Ewing) (Acari: Tetranychidae) on rice in Iran. Proceeding of the $12^{\text {th }}$ Iranian Plant Protection Congress 2 -7 Sep. 1995 Karadj P, 79.

Ghamry, E. M.; Kokab, Y. Amer and B. M. Wilson (1994): Screening test of some insecticides against the two land snails Monacha contiana and Eobania vermiculata in Sharkia Governorate. Zagazig. J. Agric. Res., 21 (5): 1539 - 1545.

Hosny, M.M., A.H. Amin and G.B. El-Saadany (1972): The damage threshold of the red scale, Aonidiella aurantii (Mask) infesting mandarin trees in Egypt. Bull. Soc. Entomol., Egypt, 71: 286-296.

Ibrahim, M. M. A. (1994): Ecological and biological studies on some pests of solanaceous plants at
Sharkia Governorate, M. Sc. Thesis, Fac. Agric. Zagazig University, 200 pp.

Ibrahim, M.M.A. (2001): Studies on some piercing sucking insect pests infesting cotton plants. Ph.D. Thesis, Fac. of Agric. Zagazig Univ.198 pp.

Ibrahim, M. M. A.; H. E. Megahed and O. M. O. Mohamed (2007): Susceptibility of three wheat cultivars to mite infestation and some mite control measurements in wheat fields at Sharkia Governorate. J. Prod. and Devl. 12 (2): 689-699p.

Krid, C. F. and F. D. Toit (1988): The brown wheat mite. Farming in South Africa, G 8:2.

Little, T. M. and F. J. Hills (1975): Statistical methods in agricultural research available from U.C.D. Book store, Univ. of California, Davis: 241ppj

Noorbakhsh, S. H. (1993): Population dynamic of brown wheat mite Petrobia latens (Muller) in western Chahar-Mahal and Bakhtiari Iran. Proceedings of the plant protection congress of Iran 28 Aug. - 2 Sep. 1993 Rasht P. 18.Pedigo, L. P.; S. H. Hutchins and L. G. Highly (1986): Economic injury levels in theory and practice. Ann. Rev. Entomol. 31: 341-368.

Prinsloo, G. (2001): When must Petrobia latens a pest on wheat be sprayed wheat focus (South Africa) V 19(5): 10 -11.

Salem, S. A. and Zaki, F. N. (1985): Economic threshold level of the citrus wax scale Ceroplastes floridensis on citrus. Egypt. Bull. Soc. Ent. Egypt, 05, 1984-1985 (333-344) p.

Stern, V. M.; R. F. Smith; R. V. D. Bosch and K. S. Hagen (1959): The integrated control concept. Hilgardia, 29: 81-101.

Wang, X. Y.; J. L. Wang and S. J. Li (1994): Study on the population dynamics and chemical control of Petrobia latens (Muller). Plant Protection. 20(3): $17-18$. 